\title{
A Coalitional Game Model for Heat Diffusion Based Incentive Routing and Forwarding Scheme (Work in Progress)
}

\author{
Xiaoqi Li, Wujie Zheng, and Michael R. Lyu \\ Department of Computer Science and Engineering \\ The Chinese University of Hong Kong, Shatin, N.T., Hong Kong \\ \{xqli,wjzheng,lyu\}@cse.cuhk.edu.hk
}

\begin{abstract}
We propose an incentive routing and forwarding scheme that integrates a reputation system into a monetary payment mechanism to encourage nodes cooperation in wireless ad hoc networks. For the first time in the literature, we build our reputation system based on a heat diffusion model. The heat diffusion model provides us a way of combining the direct and indirect reputation together and propagating the reputation from locally to globally. Further, we model and analyze our incentive scheme using a coalitional game, which is not the usual non-cooperative game like others. We further prove that under a proper condition this game has a non-empty stable core. From the evaluation we can see that the cumulative utility of nodes increases when nodes stay in the core.
\end{abstract}

Keywords: Coalitional Game, Incentive Routing, Heat Diffusion.

\section{Introduction}

The nature of wireless ad hoc networks is to let nodes cooperative together thus improve the connectivity of the whole network or execute some specific functions inside the network. However, nodes in this kind of networks may belong to different individuals or authorities and have their own interests. They may not want to help others forward routing and data packets, since that will cost their own energy and bandwidth. Consequently, it is necessary to provide incentive mechanisms to encourage cooperations among the nodes.

Incentive routing schemes for enforcing selfish agents to cooperate in wireless networks have been studied for years. One category of solution is using monetary incentives, either virtually or practically. Payment schemes need to be designed and usually are analyzed by game theoretic methods. In these schemes, the intermediate nodes declare their costs for forwarding packages. Then the routing protocol selects the lowest cost path (LCP) based on the declared costs. Afterwards the payments are rewarded to nodes on and sometimes off the LCP with the amount no less than their declared costs. However, a problem arises when nodes may purposely declare a higher cost to take advantage of the payment algorithms. So currently more research is focused on how to avoid cheating and achieve effective and also economic payments. 
Another category is employing reputation systems to stimulate the nodes to cooperative. The common idea of these systems is that each node in the network will monitor the behaviors of its neighbors. If the neighbors are observed for not executing some functions properly, their reputations will be decreased and they will be under the threat of being blocked from the network. The key challenges are how to combine the direct neighborhood reputations together and propagate them from locally to globally. In these systems, game theoretic methods can also be used to analyze the effectiveness of the threatening mechanism. In fact we consider that the method of using monetary incentives and reputation systems are not mutually exclusive and they can be combined together to design a more flexible incentive scheme.

On the other hand, the above schemes are usually modelled as non-cooperative games. However, in wireless networks nodes cannot perform routing and forwarding behaviors individually. They must cooperate together to complete one task, so it is natural to think about modelling the wireless network behaviors as a cooperative game.

In this paper, we are going to model the routing and forwarding procedures in wireless networks as a cooperative coalitional game with transferable payoff, and propose an incentive routing and forwarding scheme that combines the idea of payment mechanism and reputation system together. Then we analyze that the game has a non-empty core, which is a stable status in cooperative game just like the Nash Equilibrium in a non-cooperative game.

Regarding the combination of reputation and payment schemes, we first need to obtain a combined and globalized reputation, and then smoothly map this reputation value to a certain amount of payment. On the basis of it, we also need to design an incentive payment scheme integrating reputation and cost together. In the formulation of a coalitional game the key challenges are: 1) how to write the value function of the coalition which represents the collective payoff of the coalition; 2) how to find the solution of this game where every node has a satisfying payoff share, so that it will not deviate from some stable status. Some games may not have such a stable solution. The objective of our paper is to solve the above questions.

We list our major contributions as follows: First, we design an incentive routing and forwarding scheme that integrates reputation information into a payment mechanism. Second, we introduce a heat diffusion model to combine the direct and indirect reputations together and propagate them from locally to globally in the way how heat diffuses. Third, unlike others, we model this incentive scheme using a coalitional game method. A characteristic value function of the coalition is designed, and we prove that this game has a core solution.

The rest of this paper is organized as follows. We first give the background of the heat diffusion model in Section 2, After describing some technical preliminaries in Section 3 we will propose our incentive routing and forwarding scheme in Section 4. The scheme is analyzed in Section 5, then we show some evaluation results in Section 6. In the end, some related work and conclusions are given in Section 7 and Section 8 respectively. 


\section{Background of Heat Diffusion Model on Weighted Directed Graph}

\subsection{Motivation}

A reputation system usually needs to address two problems: 1) how to combine subjective direct reputations with indirect reputations from neighbors to make them become more objective; and 2) how to propagate the reputation from locally to globally. Previously there are different solutions to these problems such as [1] and 2. In this work, we will employ the heat diffusion model to fulfill the requirements.

In nature, heat always flows from high temperature positions to low temperature positions via conductive media. A heat diffusion model describes this phenomenon that heat can diffuse from one point to another through an underlying manifold structure in a given time period. The higher the thermal conductivity of the medium, the easier the heat flows, which implies that the two end points have some cohesive relations. Diffusion behaviors are also affected by the underlying geometric structures. Some achievements have been made based on the heat diffusion model such as classification in machine learning field, page ranking in information retrieval [3] and marketing candidates selection in social computing 4], but to our best knowledge, there is no previous work that has been performed on the incentive routing in wireless networks.

We see that in the process of heat diffusion, each node's heat comes from all of its incoming links and diffuses out to its successors as long as it can. If we diffuse heat on a weighted directed graph, the amount of heat a node can get depends not only on the heat of its neighbors but also on the weights of the links connecting them. The higher the weight, the more thoroughly the heat can be diffused. Therefore, if we let the weight be the direct reputation value of the link, then the amount of heat will be the overall reflection of the underlying reputation information. The course of heat diffusion through all possible links can also be deemed as a propagation of the reputations.

\subsection{Heat Diffusion on Weighted Directed Reputation Graph}

We construct a heat diffusion model on the reputation graph $G=(M, E, R)$, where $M=\{1,2, \ldots, m\}$ is the node set. $E=\{(i, j) \mid i$ and $j$ are in communication range and the transmission direction is from $i$ to $j\}$. The heat only flows from $i$ to $j$ if $(i, j) \in E$. $R$ is the reputation set $\left\{r_{i j} \mid r_{i j}\right.$ is the direct reputation of edge $(i, j)\}$. We use $f_{i}(t)$ to describe the heat value of node $i$ at time $t$, beginning from an initial distribution of heat $f_{i}(0)$ at time zero. $\boldsymbol{f}(t)$ denotes the vector consisting of $f_{i}(t)$.

The heat diffusion modelling is as follows. Suppose, at time $t$ node $i$ diffuses $H_{D}(i, t, \Delta t)$ amount of heat to its subsequent nodes. We assume that: a) the heat $H_{D}$ is proportional to the time period $\Delta t$; b) $H_{D}$ is proportional to the heat of node $i$; ) each node has the same ability to diffuse heat; and d) node $i$ intends to distribute $H_{D}$ uniformly to each of its subsequent nodes, but the actual 
heat it can diffuse is proportional to the corresponding reputation weight of the edge. On the basis of the above considerations, we state that node $i$ will diffuse $\lambda p_{i k} f_{i}(t) \Delta t / l_{i}$ amount of heat to each of its subsequent node $k$, where $l_{i}$ is the outdegree of node $i$ and $\lambda_{j}$ is the thermal conductivity, which is the heat diffusion coefficient representing the heat diffusion ability. In the case that the outdegree of node $i$ is zero, we assume that this node will not diffuse heat to others. Then the total amount of heat node $i$ will diffuse is $\sum_{k:(i, k) \in E} \lambda p_{i k} f_{i}(t) \Delta t / l_{i}$.

On the other hand, each node $i$ receives $H_{R}(i, j, t, \Delta t)$ amount of heat from $j$ during a period of $\Delta t$. We also have the following assumptions: a) $H_{R}$ is proportional to the time period $\Delta t$; b) $H_{R}$ is proportional to the heat of node $j$; c) $H_{R}$ is zero if there is no link from node $j$ to $i$. Based on the above considerations, we obtain $H_{R}(i, j, t, \Delta t)=\lambda_{j} f_{j}(t) \Delta t$. As a result, the heat that node $i$ receives between time $t$ and $t+\Delta t$ will be equal to the sum of the heat flowing from all its neighbors pointing to it, which is $\sum_{j:(j, i) \in E} \lambda_{j} f_{j}(t) \Delta t$. Since the amount of heat that $j$ diffuses to $i$ should be equal to the amount $i$ receives from $j$, we have $\lambda p_{j i} f_{j}(t) \Delta t / l_{j}=\lambda_{j} f_{j}(t) \Delta t$. So we get $\lambda_{j}=\lambda p_{j i} / l_{j}$. To sum up, the heat difference at node $i$ between time $t$ and $t+\Delta t$ will be the amount of heat it receives deduced by what it diffuses. The formulation is therefore:

$$
f_{i}(t+\Delta t)-f_{i}(t)=\lambda\left(\sum_{j:(j, i) \in E} \frac{p_{j i}}{l_{j}} f_{j}(t)-\mu_{i} \sum_{k:(i, k) \in E} \frac{p_{i k}}{l_{i}} f_{i}(t)\right) \Delta t,
$$

where $\mu_{i}$ is a flag to identify whether node $i$ has any outlinks. If node $i$ does not have any outlinks, $\mu_{i}=0$; otherwise, $\mu_{i}=1$. To find a closed form solution to Eq.(1), we then express it in a matrix form:

$$
\begin{gathered}
\\
\frac{\boldsymbol{f}(t+\Delta t)-\boldsymbol{f}(t)}{\Delta t}=\lambda \boldsymbol{H} \boldsymbol{f}(t), \text { where } \\
H_{i j}= \begin{cases}p_{j i} / l_{j}, & (j, i) \in E, \\
-\left(\mu_{i} / l_{i}\right) \sum_{k:(i, k) \in E} p_{i k}, & i=j, \\
0, & \text { otherwise. }\end{cases}
\end{gathered}
$$

Solving the above equation, we get

$$
\boldsymbol{f}(t)=e^{\lambda t \boldsymbol{H}} \boldsymbol{f}(0)
$$

The matrix $e^{\lambda t \boldsymbol{H}}$ is called the diffusion kernel, showing that the heat diffusion process continues infinite times from the initial heat diffusion step.

\section{Technical Descriptions}

Before presenting our incentive scheme and coalitional game we first give some technical notations. Our game is based on the bi-directional weighted graph $G=(M, E, P)$ described in Section 2 Suppose that $s$ is the source node and 


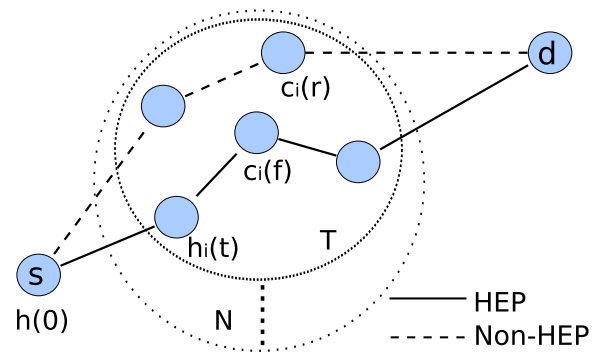

Fig. 1. Illustration of Notations of the Coalitional Game

$d$ is the destination node, then the player set of this coalitional game is $N=$ $M \backslash\{s, d\}$. Coalition is denoted by any non-empty subset $T \subseteq N$, and the overall payoff of the coalition is denoted by $v(T) \in \mathbb{R}$. Then the game is expressed by $\Gamma=\langle N, v\rangle$. Players will form into coalitions to help establishing the highest effective path between $s$ and $d$ with the lowest cost under the constraint that each intermediate node's heat is higher than a threshold $\theta$. If there's a tie in the total cost, $s$ will break the tie by choosing the path with the highest heat. The source can freely choose the value of $\theta$ to meet its requirement on reputation. The larger the value $\theta$ is, which means the source has a higher demand on reputations, the higher payments it will expend. All the paths established inside the coalition $T$ connecting $s$ and $d$ compose the path set $P_{s d}(T)$.

Initially, $s$ will load a certain amount of initial heat $f(0)$ and diffuse it on the reputation graph, then at time $t$, each node will be diffused $f_{i}(t)$ amount of heat. Correspondingly each source $s$ has an initial balance of $h(0)$, and the payment to each node $h_{i}(t)$ is paid by it according to $f_{i}(t)$. Every node evolving in the routing or forwarding procedure will cost its energy. Since the cost for sending/receiving routing and data packets are different [5], and the cost for data transmission is usually larger than that of routing packets transmission, we denote the routing and forwarding cost respectively by $c_{i}(r)$ and $c_{i}(f) \in \mathbb{R}^{+}$, and $c_{i}(r)<c_{i}(f)$ for all $i \in N$. Please see Fig. 11 for the illustration of notations.

\section{Incentive Routing and Forwarding Scheme}

The basic idea of achieving incentives is that nodes will be paid when they help others forwarding data or routing packets. Unlike other payment schemes that reward the nodes according to their claimed cost, our incentive routing and forwarding scheme pays the nodes by their reputations. The higher a node's reputation is, the higher payment it can get. The payment is given by the source node. The payment may be in the form of virtual currency like [6] or any other practical form. In our paper we assume that there is such a payment form and a payment operation daemon in the network.

In the scheme, the source node $s$ will originate the heat diffusion process starting from itself. Then after collecting the forwarding cost of all the 


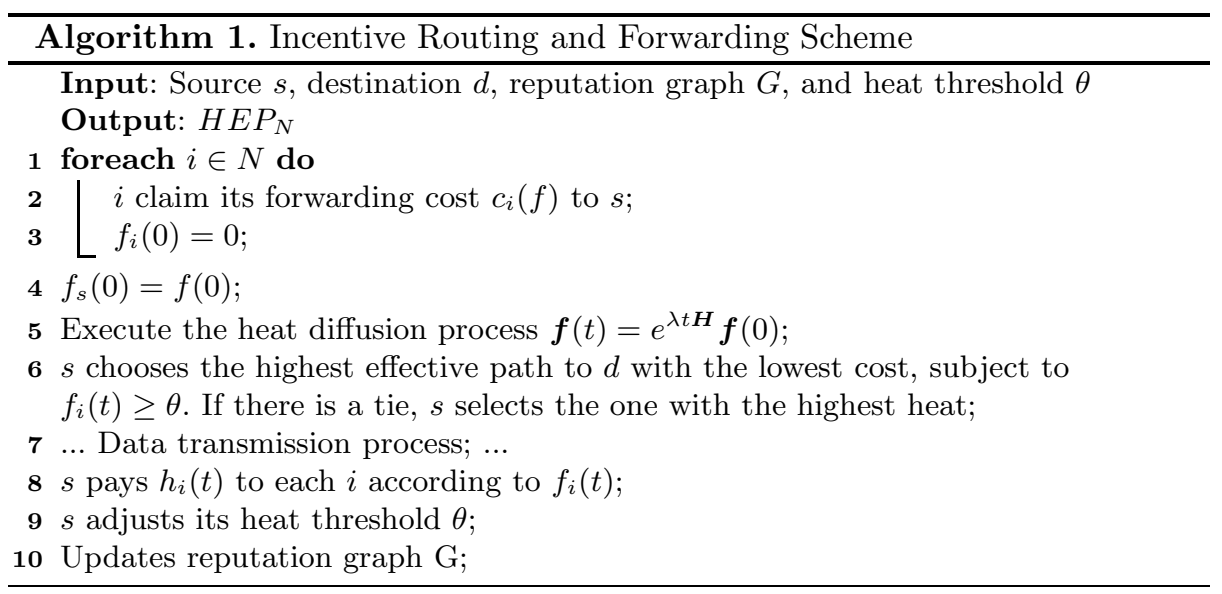

players, $s$ will compute the lowest cost path under the constraint that the diffused heat of each intermediate node is higher than its assigned threshold $\theta$. We call this path as the highest effective path (HEP). Selfish or unreliable nodes will be degraded with respect to their direct reputations by their neighbors while enthusiastic or reliable nodes will be upgraded in their reputations. The extent of increasing or decreasing a node's reputation depends on the functions it takes. Forwarding data packets will get higher reputation increments than forwarding routing packets. Correspondingly, not forwarding data packets will get heavier punishment on reputation than not forwarding routing packets. The utility a node gets in one session is the amount of payment it receives from the source node, subtracted by the cost it expends for forwarding data or routing packets. The scheme is summarized in Algorithm 1.

Under the effect of the algorithm, we can see that by behaving cooperatively a node can get higher and higher reputations, thus the payment to it will also be increased, so as to the individual utility. To earn more utility, the node will then try to improve its reputation by actively forwarding for others.

Sometimes for one session a node's utility obtained for forwarding routing packets may be higher than that of forwarding data packets. But the increasing acceleration of the latter is larger than that of the former because of the different updating way of reputation. So in the long run the cumulative utility of the node in the latter will exceed that in the former. If a node declare a higher cost than its actual forwarding cost to avoid being selected in the HEP, it will suffer the same situation. The above are some intuitive thoughts behind the scheme; for precise analysis we give it in Section 5

\section{Our Coalitional Game}

In this section we will analyze the proposed incentive scheme by modelling the routing and forwarding procedure as a cooperative coalitional game with transferable payoff. Furthermore, we show that the game has a non-empty core. 


\subsection{Value Function of the Coalition}

The value or characteristic function is the key component of a coalitional game. For each coalition $T$, the value $v(T)$ is the total payoff that is available for division among the members of $T$. It can also be interpreted to be the most payoff that the coalition $T$ can guarantee independent of the behavior of the coalition $N \backslash T[7$. Now we will define the value function of the coalition in our game. As described in Section $3, s$ is the source node and $d$ is the destination node. The player set is $N=M \backslash\{s, d\}$. When nodes join together into one coalition, they will establish one or more paths between $s$ and $d$, each of which gives the coalition a collective payoff $w_{P}(T)$, where $P \subseteq T$ represents a path. The collective payoff comes from each member's contributions of their reputation-based payments $h_{i}$, then subtracted by the costs they bear for performing routing or forwarding behaviors. For those who are only involved in the routing discovery process the cost is $c_{i}(r)$, and for those who have been selected in a path the cost should be $c_{i}(f)$. So for each path $P \subseteq T$, the corresponding payoff function for the coalition is $w_{P}(T)=\sum_{i \in T} h_{i}-\sum_{i \in P} c_{i}(f)-\sum_{i \notin P} c_{i}(r)$. Among all these payoffs, we say that the characteristic worth or the value of the coalition $v(T)$ should be the maximal collective payoff it can guarantee, which is:

$$
v(T)=\max _{P \subseteq T}\left(\sum_{i \in T} h_{i}-\sum_{i \in P} c_{i}(f)-\sum_{i \notin P} c_{i}(r)\right) .
$$

We call the path that has the maximal $w_{P}(T)$ as the highest effective path $H E P_{T}$, so alternatively we can write $v(T)=\sum_{i \in T} h_{i}-\sum_{i \in H E P_{T}} c_{i}(f)-$ $\sum_{i \notin H E P_{T}} c_{i}(r)$. But if there is no such path inside the coalition, the coalition is inessential and worths nothing, and the value of it is 0 . Then formally, we have the definition of $v(T)$ as follows.

Definition 1 (Value Function of A Coalition). The value of any coalition $T \subseteq N$ is 0 when there is no path between $s$ and $d$ inside $T$. That is: $v(T)=0$, if $\bar{P}_{s d}(T)=\phi$. Otherwise, $v(T)$ is:

$$
v(T)=\sum_{i \in T} h_{i}-\sum_{i \in H E P_{T}} c_{i}(f)-\sum_{i \notin H E P_{T}} c_{i}(r), \quad \text { if } P_{s d}(T) \neq \phi
$$

\subsection{Nonemptiness of the Core}

The key issue of a cooperative game is regarding how to divide earnings inside the coalition in some effective and fair way. The adequate allocation profile is then called a solution, which is a vector $\boldsymbol{x} \in \mathbb{R}^{N}$ representing the allocation to each player when a grand coalition is formed. The grand coalition means all the players form into one coalition. The core is one of the solution concepts for cooperative games. If a coalitional game's core is non-empty, it means that no coalition can obtain a payoff that exceeds the sum of its members' current payoffs, which 
means no deviation is profitable for all of its members [7]. Theoretically the core is the set of imputation vectors which satisfies the following three conditions:

1. $x(i) \geq v(i)$

2. $x(T) \geq v(T), \quad \forall T \in 2^{N}$

3. $x(N)=v(N), N$ is the player set

where $x(i)$ is the payoff share of node $i$ in this game, $x(T)=\sum_{i \in T} x(i)$, and $x(N)=\sum_{i \in N} x(i)$.

The core of a coalitional game is possibly empty. Next we will analyze in which condition our game has a non-empty core, and what the possible core is. We derive the following theorem.

Theorem 1. Under the condition of $h_{i} \geq c_{i}(f)$ for each player $i$, the payoff profile $x$ is in the core of the coalitional game where

$$
x(i)= \begin{cases}h_{i}-c_{i}(f), & i \in H E P_{N} \\ h_{i}-c_{i}(r), & i \notin H E P_{N}\end{cases}
$$

Proof. Firstly, check the first requirement of Eq.7. Under the condition of $h_{i} \geq$ $c_{i}(f)$, we have $x(i)=h_{i}-c_{i}(f) \geq 0$. When the coalition has only one member $i$, the value of it would be 0 if $i$ cannot establish a path between $s$ and $d$. That is $v(i)=0$. Thus $x(i) \geq v(i)$ holds. If $i$ can connect $s$ and $d$, then $v(i)=h_{i}-c_{i}(f)$ as said by Def 1 . In that case $x(i)=v(i)$ which also meets the first requirement.

Secondly, from Eq[8] and Def[1, we have $x(N)=\sum_{i \in N} x(i)=\sum_{i \in H E P_{N}}\left(h_{i}-\right.$ $\left.c_{i}(f)\right)+\sum_{i \notin H E P_{N}}\left(h_{i}-c_{i}(r)\right)=\sum_{i \in N} h_{i}-\sum_{i \in H E P_{N}} c_{i}(f)-\sum_{i \notin H E P_{N}} c_{i}(r)=$ $v(N)$. So the third requirement of Eq[7 also holds.

Thirdly, to prove $\boldsymbol{x}$ satisfies the second requirement $x(T) \geq v(T)$, we will list and analyze all of the different HEP situations in the grand coalition $N$ and an arbitrary coalition $T$. For those coalitions without paths inside, the values of them are 0 , so we easily get $x(T) \geq v(T)=0$. For other coalitions, there are totally four kinds of situations as illustrated in Fig 2 .

The proof for these four situations are similar. Because of the space limit, we only prove the most complicated situation in Fig $2(\mathrm{~d})$ here. In this case, when the grand coalition $N$ is formed, the new $H E P_{N}$ is different from $H E P_{T}$ and part of $H E P_{N}$ is inside $T$. For clarity we first give the following notations for Fig.2(d). We let $A=H E P_{N} \cap T, B=H E P_{N} \cap(N \backslash T), C=H E P_{T} \cup A$,

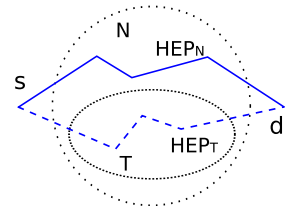

(a)

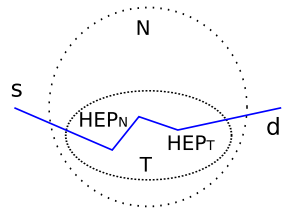

(b)

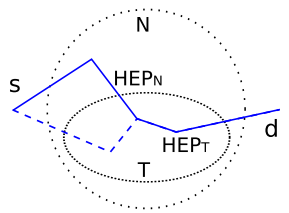

(c)

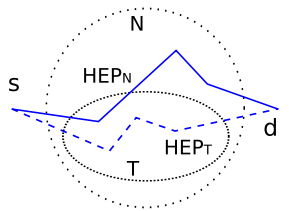

(d)

Fig. 2. Examples of Different HEP Situations 
$D=T \backslash C$, and $E=N \backslash\left(H E P_{N} \cup H E P_{T}\right)$. According to Def[1 and Eq 8 we have:

$$
x(T)-v(T)=\left[\sum_{i \in H E P_{T}} c_{i}(f)-\sum_{i \in H E P_{T}} c_{i}(r)\right]-\left[\sum_{i \in A} c_{i}(f)-\sum_{i \in A} c_{i}(r)\right]
$$

$H E P_{N}$ and $H E P_{T}$ are two paths connecting $s$ and $d$ inside the grand coalition $N$, and $H E P_{N}$ dominates $H E P_{T}$. So based on Eq 5 , we have $v_{H E P_{N}}(N) \geq$ $v_{H E P_{T}}(N)$. Through deduction we get:

$$
\begin{aligned}
& \sum_{i \in H E P_{T}} c_{i}(f)-\sum_{i \in H E P_{T}} c_{i}(r) \geq\left[\sum_{i \in A} c_{i}(f)-\sum_{i \in A} c_{i}(r)\right]+\left[\sum_{i \in B} c_{i}(f)-\sum_{i \in B} c_{i}(r)\right] \\
& >\sum_{i \in A} c_{i}(f)-\sum_{i \in A} c_{i}(r)
\end{aligned}
$$

Then substitute in Eq9, we get $x(T) \geq v(T)$. So the second requirement is satisfied. In summary, under the condition of $h_{i} \geq c_{i}(f)$ for each player $i$, the proposed payoff profile $\boldsymbol{x}$ is in the core of this coalitional game.

We can see that if only the payment a node gets based on its reputation is larger than the cost it needs to forward data packets, the core of this coalitional game exists. Nodes who want to get more payoff share $x_{i}$ must try to improve its reputation by helping others forwarding or increasing its link reliability, so that it can get more diffusion of the heat-based payment. In this way a virtuous cycle can be created.

\section{Evaluations}

We have theoretically proved that our incentive scheme guarantees the existence of the core when modelled as the coalitional game. Now we will evaluate the scheme in two aspects through experiments: 1) how is the general overview of all the nodes' utility and how does the network topology affect the distribution of it; and 2) how the nodes' cumulative utilities and balances evolve over time.

We conduct the evaluation on a randomly generated wireless topology with 100 nodes scattering in an area of 3000 by 3000 meters. The radio range is set to 422.757 meters. The topology is shown in Fig 3(a) There is a line connecting two nodes when they are in the communication range of each other. We label some representative nodes for further illustration. Each node has an initial balance of 100 and each directed link has a local reputation value as the weight. At each round we randomly select a source-destination pair and the source $s$ perform the incentive routing and forwarding algorithm. We assign the parameter $\lambda$ in 


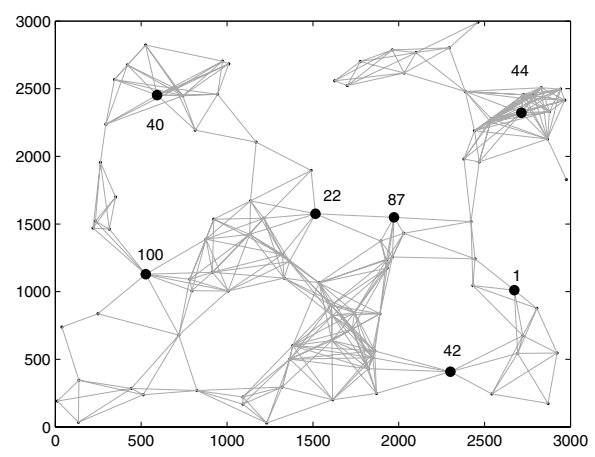

(a)

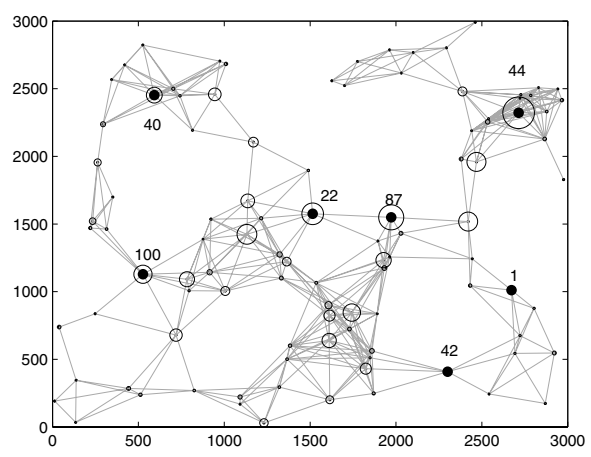

(b)

Fig. 3. Network Topology and Overview of Nodes' Utilities

the heat diffusion equation as 1 . The evaluation runs for 1000 seconds and we observe the utility and balance of each node every second.

Our first evaluation shows the overview utility at the end of the experiment in Fig $3(\mathrm{~b})$, The circles around the nodes represent the cumulative utility of that node. The diameter of the circle is proportional to the amount of the utility.

We observe that in general nodes in the high density area also have large circles around them (like node 44), and on the contrary, nodes in the sparse area usually have indistinctive circles.

Our second evaluation starts from the core of the coalitional game. Fig 4(a) and 4(b) show the cumulative utilities and balances of several typical nodes respectively over the simulation time. The balance of a node may fall below the initial balance (like node 42 ) because the nodes have their own data transmission requests and what they earn cannot compensate what they pay.

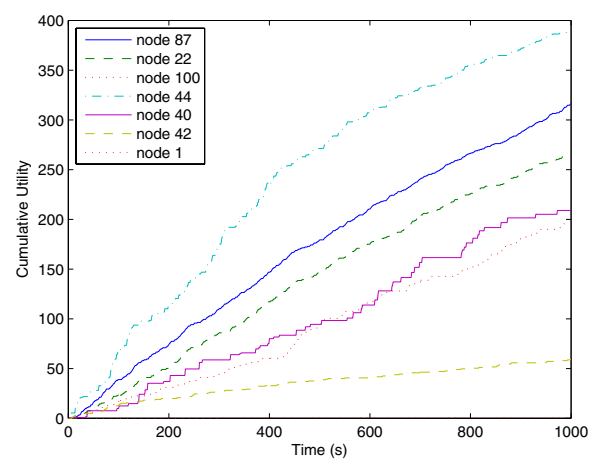

(a)

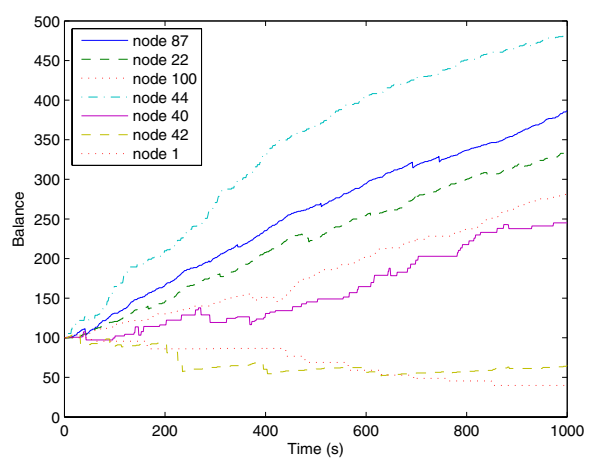

(b)

Fig. 4. Cumulative Utility and Balance of Nodes as a Function of Simulation Time 


\section{Related Work}

Many incentive routing schemes have been proposed in the past few years. Most approaches fall into one of two main categories. In the first category, nodes forwarding packets get monetary incentives for their service. In Ad Hoc-VCG 8, payments are paid to nodes consisting the actual costs incurred by forwarding data and the extra premiums. The implemented reactive routing protocol is a variation of the well-known VCG mechanism. It achieves the design objectives of truthfulness and cost-efficiency in a game-theoretic sense. Another work [6] introduces a virtual currency called nuglets. The source of the packet must load it with enough nuglets to pay for the trip to the destination. Cooperation is enforced in this scheme because nodes must forward packets for others in order to build up enough nuglets to get their own packets forwarded. 9 designs an incentive-compatible routing and forwarding protocol integrating VCG mechanism and cryptographic technique. Payments are implemented based on VCG protocol and the application of cryptographic techniques in the design of forwarding protocol enforces the routing decision. [10] designs a collusion-resistant routing scheme for non-cooperative wireless networks. Payments are given to nodes not only on the LCP paths but also off the paths.

In the second category, non-cooperative nodes are identified based on a reputation system and circumvented in the routing process. In CORE [1], node cooperation is stimulated by a collaborative monitoring technique and a reputation mechanism. Each node of the network monitors the behavior of its neighbors with respect to a requested function and collects observations about the execution of that function. CONFIDANT [11] differs from CORE only in that it sends reputation values to other nodes in the network, which exposes the scheme to malicious spreading of false reputation values. Liu and Issarny employ a Bayesian approach to design an incentive compatible reputation system to facilitate the trustworthiness evaluation of nodes [12. Some also use subjective logic to calculate uncertain trust so as to design secure routing protocols [2] or incentive reputation mechanisms [13].

\section{Conclusion and Future Work}

In this paper, we present a novel incentive routing and forwarding scheme which combines reputation system and payment mechanism together to encourage nodes to cooperate in wireless ad hoc networks. Besides, we design our reputation system based on a heat diffusion model for the first time in the literature. The heat diffusion model provides us a way of combining the direct and indirect reputations together and propagating the reputation from locally to globally. Further, instead of using the non-cooperative game method, we model and analyze our incentive scheme using a coalitional game. We further prove that under a certain condition this game has a non-empty core. Through the evaluation we can see that the cumulative utility of nodes increases when the nodes stay in the core. In the future we will consider to apply other underlying reputation systems to our incentive scheme. 


\section{Acknowledgement}

The work described in this paper was fully supported by a grant from the Research Grants Council of the Hong Kong Special Administrative Region, China (Project No. CUHK4158/08E).

\section{References}

1. Michiardi, P., Molva, R.: Core: a collaborative reputation mechanism to enforce node cooperation in mobile ad hoc networks. In: IFIP CMS, Deventer, The Netherlands, pp. 107-121. Kluwer, B.V, Dordrecht (2002)

2. Li, X., Lyu, M.R., Liu, J.: A trust model based routing protocol for secure ad hoc networks. In: Proceedings of IEEE Aerospace Conference, pp. 1286-1295 (March 2004)

3. Yang, H., King, I., Lyu, M.R.: Diffusionrank: a possible penicillin for web spamming. In: SIGIR, pp. 431-438. ACM, New York (2007)

4. Ma, H., Yang, H., Lyu, M.R., King, I.: Mining social networks using heat diffusion processes for marketing candidates selection. In: CIKM, October 2008, pp. 233-242 (2008)

5. Feeney, L.M., Nilsson, M.: Investigating the energy consumption of a wireless network interface in an ad hoc networking environment. In: INFOCOM, vol. 3, pp. 1548-1557 (2001)

6. Buttyan, L., Hubaux, J.P.: Nuglets: a virtual currency to stimulate cooperation in self-organized mobile ad hoc networks. Technical Report DSC/2001/001, Dept. of Communication Systems, Swiss Federal Institute of Technology - Lausanne (2001)

7. Osborne, M.J., Rubinstein, A.: A Course in Game Theory. The MIT Press, Cambridge (1994)

8. Anderegg, L., Eidenbenz, S.: Ad hoc-vcg: A truthful and cost-efficient routing protocol for mobile ad hoc networks with selfish agents. In: MobiCom, pp. 245259. ACM, New York (2003)

9. Zhong, S., Li, L.E., Liu, Y.G., Yang, Y.R.: On designing incentive-compatible routing and forwarding protocols in wireless ad-hoc networks - an integrated approach using game theoretical and cryptographic techniques. In: MobiCom, pp. 117-131. ACM, New York (2005)

10. Zhong, S., Wu, F.: On designing collusion-resistant routing schemes for noncooperative wireless ad hoc networks. In: MobiCom, pp. 278-289. ACM, New York (2007)

11. Buchegger, S., Boudec, J.Y.L.: Performance analysis of the confidant protocol: Cooperation of nodes - fairness in dynamic ad-hoc networks. In: MobiHoc, Lausanne, CH (June 2002)

12. Liu, J., Issarny, V.: An incentive compatible reputation mechanism for ubiquitous computing environments. IJIS 6(5), 297-311 (2007)

13. Kane, K., Browne, J.C.: Using uncertainty in reputation methods to enforce cooperation in ad-hoc networks. In: WiSe 2006: Proceedings of the 5th ACM workshop on Wireless security, pp. 105-113. ACM, New York (2006) 